\title{
Energy Optimization using Neighborhood based Weighted Rendezvous Technique for Wireless Sensor Networks
}

\author{
Ruthvic S D \\ PG Scholar \\ Dept. of CSE \\ NMAMIT, Nitte, India.
}

\author{
Ravi B \\ Research Scholar \\ Dept. of CSE \\ REVA University, India.
}

\author{
Udaya Kumar Shenoy \\ Professor \\ Dept. of ISE \\ NMAMIT, Nitte, India.
}

\begin{abstract}
Establishing an energy efficient wireless sensor network is a challenging task. A significant amount of research work has already been carried out in this direction to study energy optimization in WSN by taking the advantage of mobile sink or mobile agents. Many approaches using mobile sink have demonstrated that energy usage can be optimized significantly in the phenomenon area from where the sink would collect the sensed readings from the sensor nodes via single or multi hop communication. But, the slow mobility speed of the sink will tend to increase the data collection latency in the sensor network, especially in delay bound WSN applications. To overcome this problem, several rendezvous based techniques have been proposed, in which sink is allowed to visit a subset of the sensor nodes to collect the data via single hop communication. This subset of nodes, called rendezvous points (RPs), is considered as data collection points. All other nodes send their sensed data using the shortest path to these RPs. In this paper a simple neighbourhood based rendezvous technique is proposed. In our approach a subset of sensor nodes from the network is designated as rendezvous points (RPs) set to receive and buffer the data from their nearest source nodes. The selected RP set is such that they take care of denser part of the network where energy consumption is more so that the energy hole problem can be minimized and help optimize the energy consumption in the network. A shortest sink tour is then constructed using only RPs, using which mobile sink makes its tour and collects the buffered data from the RPs within a given deadline. In this paper we explain the NBWRP (Neighbourhood based Weighted Rendezvous Planning) algorithm to compute the RPs. We also evaluate and compare its performance with static sink WSN and WSN with random sink mobility. Our results show that the algorithm achieves better WSN lifetime compared to static sink case and random movement strategy.
\end{abstract}

\section{General Terms}

Wireless Sensor Networks, Mobile base station.

\section{Keywords}

Wireless Sensor Network (WSN), Mobile Sink, Controlled Mobility, Rendezvous Point, Energy optimization.

\section{INTRODUCTION}

Wireless Sensor Networks (WSNs) consist of hundreds or thousands of sensor nodes, which can be deployed in a remote hostile environment to achieve specific objectives. WSNs play an important role in a wide range of applications, such as emergency response [13], environment monitoring [11], smart transportation [12], routine data collection [15], and hazard detection [14]. Sensor nodes in a WSN sense the phenomenon and cooperatively forward the sensed data to the sink or base station via multi-hop wireless communication. A sink, which may be static/mobile, acts like a connection between users and the network. One can get the required information from the network by sending the queries and collecting results from the sink. The sensor nodes can also communicate among themselves using radio signals. Each sensor node is equipped with computing and sensing devices, power components and radio transceivers. Also each sensor node in a wireless sensor network (WSN) is resource constrained. i.e., they have limited processing speed, battery power, storage capacity and communication bandwidth [1]. In many applications replacing sensor node's batteries is difficult or impractical. Therefore it is very important to efficiently utilize sensor node's energy to accomplish a specific mission.

In WSN with a static sink, the sensed readings of the sensors

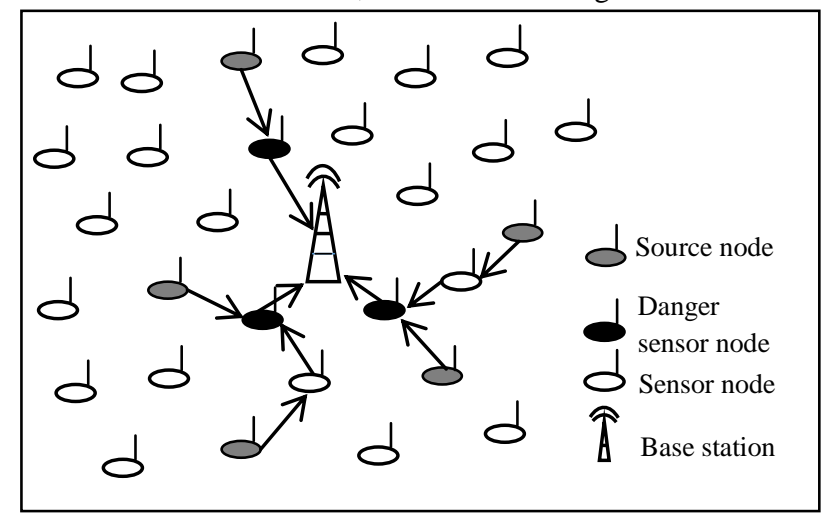

Fig. 1: The phenomenon of energy hole problem in wireless sensor network with a static sink.

are usually routed via multihop forwarding to the sink for processing. This type of communication creates a bottleneck in the sensor network. That is, the nodes located near the sink are overloaded since they need to forward more data from the nodes located farther away from the sink. Therefore, the battery of these nodes depletes earlier than that of other far away sensor nodes. This leads to non-uniform energy distribution of the nodes which finally results in network partition $[1,2]$. This phenomenon is known as "energy hole" or "hot spot" problem [1] which is shown in Fig. 1.

Several research studies have shown that the usage of mobile sink can alleviate "hot spot" problem and hence improve 
energy optimization of the network by gathering the readings directly from the sensor nodes when the sink moves to the node's vicinity. The data collection can occur via single or limited multi hop communication. The sink movement is either controlled or uncontrolled [3]. Data MULEs explained in [16] are a good example for uncontrolled sink mobility. In this a number of MULEs move throughout the network to provide one-hop data collection and improve the network connectivity. Uncontrolled sink mobility is advantageous in delay tolerant application in which there is a trade-off between data collection latency and energy consumption. Since the proposed technique is for delay-sensitive applications, we use controlled sink mobility in which the movement of sink is controlled by several network parameters such as delay, current network energy, node density etc. In this approach the sink plans its tour based on the application deadline, locations of RPs and available energy in the network.

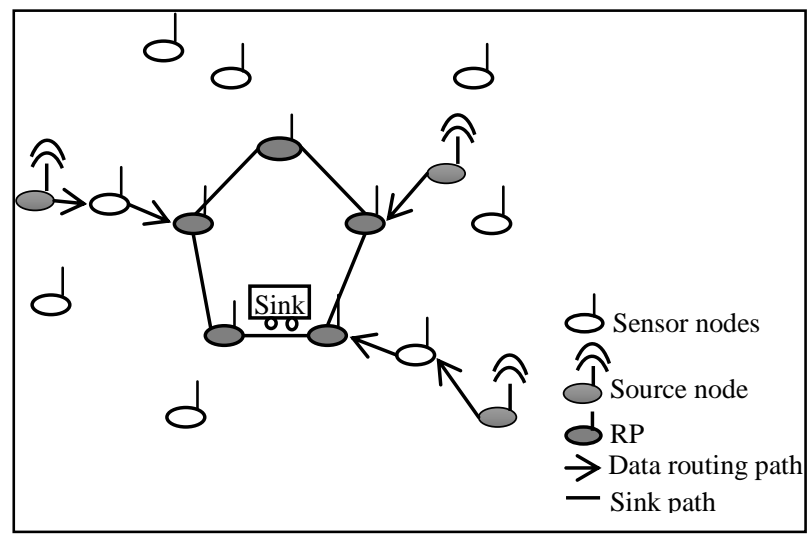

Fig. 2: The scenario of rendezvous based technique.

In WSN with a mobile sink, one principle problem is to determine how we can move the sink [7] to collect the sensed data from the network. One approach is to visit each sensor node to receive the sensed data directly via single hop communication. This can be formulated as Travelling Salesman Problem (TSP) which is a well-known NP-hard problem in graph theory. The objective of the TSP is compute a tour for the salesman to visit a set of cities such that the total tour cost is minimized and that each city is visited exactly once by the salesman. Many heuristic approaches have been proposed in the literature to achieve near optimal solution for TSP. However, when used in WSN with a mobile sink, these heuristic solutions become impractical as the number of nodes increases [4]. Moreover, typical speed of mobile sink is slow and therefore it increases data collection latency in a large network $[5,23]$. In this paper, we propose a rendezvous based technique in which a subset of sensor nodes is selected as Rendezvous Points (RPs). Other sensor nodes forward the sensed data to their nearest RP using a shortest path and the sink collects the cached data at each RP via single hop communication by making the tour computed using these RPs, while satisfying the timeliness constraint on data collection as shown in Fig. 2 .

The rest of the paper is structured as follows: Section 2 describes the existing methods of RP techniques. Section 3 defines the problem. In section 4, we propose the Neighbourhood Based Weighted Rendezvous Planning (NBWRP) technique. Section 5 analyses the algorithm. Section 6 presents the simulation results of NBWRP and finally section 7 concludes the paper.

\section{RELATED WORK}

Using mobile sinks for energy optimization in WSNs has been an interesting area of research and there have been many proposals in recent literature. Three major categories of mobile sink approaches are briefly explained below [10].

It has been observed that "energy hole" problem occurs when the sink stays for a long time at some point in the network. Due to multi-hop forwarding, the nodes around the sink deplete their energy earlier than all other nodes. Based on this observation several proposals [18, 19] have investigated mobile sink approach in which the sink changes its position regularly so as to change the route for forwarding data packets. Although the approach reduces the energy consumption in the network, it requires recomputation of multi-hop route every time the sink changes its position and this places a potentially prohibitive burden on the nodes.

In the second category, several approaches based on the Traveling Salesman Problem (TSP), possibly with additional constraints arising from buffer limitations in the sensor nodes, have been studied. In these approaches, the sink comes in direct contact with each sensor node and collect sensor's data via single hop, short-range communication. Several heuristics $[2,17,24]$ have been proposed to that effect so that each sensor is visited before its buffer is full. By avoiding multihop communications, this approach significantly reduces the energy consumption. But it incurs a high delay when the network area comprises a large number of nodes because of the requirement that the sink physically visit all the sensor nodes. This makes it impractical for use in delay-sensitive applications.

The last category includes the approaches that combine multihop communication with data collection by mobile sinks. The proposed method falls into this category. Several rendezvous based techniques have been investigated to deal with the problem of collecting sensed data within their deadlines. This approach is most suitable for applications with timeliness constraints on sensed data. Taxonomy provided in [6] further divides the rendezvous based approach into 3 categories: 1) RP Selection by Fixed Track 2) RP Selection by Reporting Tree 3) RP Selection by Clustering. Below we briefly explain some major rendezvous based algorithms.

A straight line sink path algorithm for data collection was proposed by Kansal et al. [21]. A number of trees, each rooted at a node along the sink path, are constructed after the initialization phase, and the root of every tree is considered as an RP to which each source sends its measurement. Xing et al. [8] presented a Minimum Spanning Tree based rendezvous design (RD-FT) for fixed track. The objective of RD-FT is to select a set of RPs along the sink track such that the total length of edges that connect sources to RPs is minimized.

Xing et al. [5, 22] proposed two algorithms by considering a geometric tree TR rooted at the base station. A node in TR is called junction node if it is shared by multiple data reporting paths in the tree. An RP could be any point on an edge of the tree. Two types of mobility are studied: constrained and unconstrained sink mobility. For the first case, the authors presented RP-CP (Rendezvous Planning with Constrained Path) which iteratively adds edges to the tour until the tour length becomes less than $\mathrm{L} / 2$, where $\mathrm{L}$ is the maximum allowed tour length. A part the next unchosen edge may be added to make it exactly L/2. For unconstrained sink mobility, a greedy heuristic algorithm called RP-UG (Rendezvous Planning with Utility-based Greedy heuristic) was presented that adds virtual nodes to geometric tree TR such that every 
tree edge is no longer than a predefined value $\mathrm{L}_{0}$. The algorithm terminates when either all the sources are added or maximum tour length is reached.

Xing et al. in [8] proposed tree based algorithm known as Rendezvous Design with a Variable BS Track (RD-VT) to compute RPs based on SMT (Steiner Minimum Tree) with the objective of decreasing the data path length from sources to their RPs. The tree is traversed in pre-order starting from the sink until the minimum distance between the visited nodes is equal to the required packet delivery time.

Khaled Almi'ani et al. in [9] proposed a cluster based algorithm with the objective to decrease the hop count from all the nodes to their respective rendezvous point. Cluster based algorithm operates in iterative manner and group the network into number of balanced size clusters. Only one RP from each cluster is selected followed by the computation of sink tour. The computed tour is such that the sink visits the maximum number of clusters while satisfying the tour length constraint. Salarian et al. [4] proposed WRP algorithm to compute RPs by assigning weights to the sensor nodes. The weights are calculated by multiplying the number of data packets forwarded and the hop distance to the closest RP on the tour. An SMT for forwarding the data is constructed for each RP in the final tour. The sink then visits these highest weighted nodes in order to reduce the energy consumption and to minimize "energy hole" problem.

\section{PROBLEM STATEMENT}

We consider a randomly deployed homogeneous WSN in which some areas are denser than other areas. In our problem the sink needs to collect the data periodically from the WSN, say every 100 seconds. First task is to compute a subset of sensor nodes to act as RPs using which the sink plans its tour and from which the sink collects the sensor node's data. The second task is to find the shortest path for each source node to send the data to its nearest RP. These two tasks need to be accomplished in such a way that when the sink moves along the tour, data should be gathered within the maximum allowed packet deadline and energy consumption should be distributed evenly across the whole WSN at any point in time. It is important to note that these tasks are interdependent and each task has a profound impact on the other. Therefore it is important to jointly optimize these two tasks.

We assume that network is connected and the sink is aware of the locations of all sensor nodes in the WSN. All the sensor nodes have a fixed communication range. We further assume that every RP has sufficient buffer space to cache all sensed data. We also assume that the communication time between sink and RPs is negligible, as compared with sink tour time. The sink moves with a constant speed and stays at each RP for sufficient amount of time to receive all cached data. In our problem each source node produces one data packet of fixed size every $t$ seconds, where $t$ is fixed for each data collection round. Finally, we assume that all data packets have the same deadline.

Formally, we consider a complete graph $G(V, E)$ representing a WSN, where $V$ is the set of homogeneous sensor nodes and $E$ is the set of edges between the nodes present in G. Let $n_{l}$, $n_{2} \ldots \ldots n_{|V|}$ be the set of randomly deployed sensor nodes, where $n_{i} \in V$. Let $R=r_{l}, r_{2, \ldots}, r m$ be the set of RPs, where $R$ $\subseteq V$. Let $d_{i j}$ be the distance between $n_{i}$ and $n_{j}$ computed using Euclidian distance formula. Let $D$ and $v$ represent the delivery deadline of packets and the constant speed at which sink moves, respectively. The objective is to find the set $\mathrm{R}$ and associated tour with the maximum allowed length $L_{\max }\left(L_{\max }\right.$

$=D \times v$ ) that visits each node in $R$ and arrives at the sink point before the deadline $D$. In other words the goal is to determine the set $\mathrm{R}$ that satisfies $L_{\max }$. It is very clear that maximum energy saving occurs when $|R|=|V|$ provided the tour satisfies $L_{\max }$. That is, energy consumption is zero when all the sensor nodes are designated as RPs with associated maximum sink tour length $L_{\max }$.

\section{Neighbourhood Based Weighted Rendezvous Planning (NBWRP)}

The aim is to select RPs from densely deployed part of the network and selection of RPs is such that the energy consumption should be evenly distributed across the network.

2

$$
3
$$

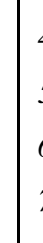$$
8
$$$$
9
$$$$
10
$$$$
11
$$$$
12
$$$$
13
$$

Input: A complete graph $G(V, E), D$ (Deadline), $v($ sink speed)

Output: $R P$ set $R=\left\{r_{1}, r_{2}, \ldots r_{n}\right\}$, where $r_{i} \in V_{U}$ Sink

begin

TotalRP $=0$, MaxWeight $=0, R P=-1, w t=0 ;$

mark $=[$ false, false .....], flag $=$ false ;

$N O N=[0,0, \ldots]$;

$R=R U$ Sink; TotalRP $=$ TotalRP $+1 ;$

$L_{\text {max }}=D \times v$;

for $i=0$ To $|V| d o$

$N O N(i)=($ number of neighbours of $i)$ +1 ;

endfor

while TotalRP $<=|V|$ do

MaxWeight $=0 ;$ flag $=$ false;

start:

for $i=0$ to $|V| d o$

$w t=N O N(i) \times \operatorname{Dist}(i, R)$;

if not mark(i) \&\& wt > MaxWeight then

$R P=i$;

MaxWeight $=w t$;

flag $=$ true

endif

endfor

if flag $==$ false then

end if break;

$\operatorname{mark}(R P)=$ true $; R=R U R P ;$

TotalRP $=$ TotalRP +1

if $\operatorname{TSP}(R) \leq L_{\max }$ then

for $i=0$ to $|V|$ do

if $i$ does not belong to $R$ then $\operatorname{mark}(i)=$ false;

endfor

else

$R=R-R P ;$ TotalRP $=$ TotalRP $-1 ;$

MaxWeight $=0$; flag $=$ false;

endif

endwhile

endbegin

Fig. 3: NBWRP Algorithm 
Since the "energy hole" problem is more likely to occur in the congested region of the network, the algorithm attempts to select RPs from this congested region. Our method is inspired by the technique proposed in [4] that uses two parameters to assign a weight to a node: the number of packets forwarded by the node and other is the hop distance to the nearest RP. A node with the highest weight, where weight is the product of the two parameters, is then selected as candidate RP. Considering this, we propose NBWRP algorithm which assigns a weight to each sensor node based on the number of its neighbours and hop distance to the nearest RP. As in [4], the algorithm selects a node with highest weight as an RP. Once all RPs are obtained, each sensor node is then associated with its nearest RP and each sensor with data uses the shortest path to forward the data to their nearest RP.

We used Christofides [20] TSP solver to construct a tour by considering the most weighted sensor nodes. The sink starts its tour by visiting only the RPs and the computed tour for the sink should be no longer than the maximum allowed tour length i.e. $L_{\max }$ As mentioned, NBWRP designates a sensor with highest weight as RP by taking two parameters into account. One is the number of neighbours of each node and its distance to the nearest RP. The weights are calculated as follows.

$$
\text { MaxWeight }=\operatorname{NON}(i) \times \operatorname{Dist}(i, R)
$$

where, $N O N(i)$ is the number of neighbours of node $i$, Dist $(i$, $R$ ) is the distance from node $i$ to the nearest RP in R.

The equation (1) clearly implies that the nodes situated far away from the selected RP or the nodes that have large number of neighbours within the communication range will be assigned with the highest weight and elected as RP. Initially, we consider the sink as being the highest weighted node followed by the RP set R is computed. If a sensor node is located one-hop away from the selected RP and has the least number of neighbours gets the lowest weight. A node without any neighbour is considered as the node with one neighbour. Hence, by visiting most weighted nodes, the algorithm attempts to reduce the multi-hop transmissions and balance the load among the sensor nodes. Thus, NBWRP results in optimizing the energy consumption and in turn improves the network lifetime. The algorithm NBWRP is given in Fig. 3.

\section{ANALYSIS OF THE ALGORITHM}

The algorithm selects all the sensor nodes as RPs when the number of neighbours of every node is zero and the sink tour meets the deadline $L_{\max }$. In this case minimum network energy consumption occurs because the sink visits every sensor node and collects data within the deadline $D$. This is similar to direct contact approach with additional constraint of satisfying $L_{\max }$. The worst case running time of the algorithm is $O\left(n^{5}\right)$, since the TSP is called for a maximum of $\mathrm{n}^{2}$ times, where $n=$ $|V|$, and the time complexity of Christofides TSP solver is $O\left(n^{3}\right)$. Therefore the run time complexity of the algorithm dependent on the number of times the TSP is called by NBWRP. Because the algorithm checks to see if every sensor can be added to the tour, it finds a tour when there is at least one possible tour in the network.

We prove that energy consumption is least when the sink visits most weighted node, as compared to visiting any other nodes. Let $n_{1}$ and $n_{2}$ be two nodes whose weights are calculated as,

$$
\begin{aligned}
& w t\left(n_{1}\right)=\operatorname{NON}\left(n_{1}\right) \times \operatorname{Dist}\left(n_{1}, R\right) \text { and } \\
& w t\left(n_{2}\right)=\operatorname{NON}\left(n_{2}\right) \times \operatorname{Dist}\left(n_{2}, R\right) \text { according to (1). }
\end{aligned}
$$

Let $w t\left(n_{1}\right)>w t\left(n_{2}\right)$. Let $E\left(n_{1}\right)$ and $E\left(n_{2}\right)$ be the energy consumed by the nodes $n_{1}$ and $n_{2}$ respectively, to send sensed data to their nearest RP using the shortest path. Then we have,

$E\left(n_{l}\right)=($ Energy consumed for transmission and reception of sensed data $) \times w t\left(n_{1}\right)$

$E\left(n_{2}\right)=($ Energy consumed for transmission and reception of sensed data) $\times w t\left(n_{2}\right)$

Since $w t\left(n_{1}\right)>w t\left(n_{2}\right)$, we have $E\left(n_{1}\right)>E\left(n_{2}\right)$. Therefore visiting $n_{l}$ will result in less energy consumption.

\section{SIMULATION RESULTS}

This section provides the performance results of NBWRP algorithm. NBWRP algorithm is implemented using NS2 simulator. We compare NBWRP with two data collection approaches, namely SS (static sink) and RSM (random sink mobility). We consider a WSN consisting of connected homogeneous sensor nodes deployed randomly in an area of $200 * 200 \mathrm{~m}^{2}$. Each experiment is repeated for 10 times over different topologies. The table-I shows the parameter list used for the simulation.

Table I. Simulation Parameters

\begin{tabular}{|l|l|}
\hline Parameter & Value \\
\hline Simulation area & $200 \mathrm{~m} * 200 \mathrm{~m}$ \\
\hline Number of Sensor nodes & 5 to 100 \\
\hline Mobile sink speed $(v)$ & $5 \mathrm{~m} / \mathrm{s}$ \\
\hline Communication range & $50 \mathrm{~m}$ \\
\hline Packet length & 512 bytes \\
\hline $\begin{array}{l}\text { Consumed energy in } \\
\text { transmitter circuit }\end{array}$ & $3.0 \mathrm{~W}$ \\
\hline $\begin{array}{l}\text { Consumed energy in } \\
\text { receiver circuit }\end{array}$ & $1.0 \mathrm{~W}$ \\
\hline $\begin{array}{l}\text { Maximum allowed packet } \\
\text { delay }(D)\end{array}$ & 100 to 200 seconds \\
\hline Sensor node's energy & $100 \mathrm{~J}$ \\
\hline
\end{tabular}

Fig. 4 shows the average network energy consumption (in joules) by NBWRP, SS and RSM for different number of nodes. Fig. 4 clearly shows that the energy consumed by NBWRP is significantly lower, as compared with energy consumed by other two approaches. SS and RSM consume almost same amount of energy. Fig. 5 shows the standard deviation of sensor node's energy consumption for different number of nodes. The standard deviation is used to measure the imbalance in the energy consumption of sensor nodes [4]. The goal is to achieve lower SD which indicates better energy optimization. The standard deviation metric is calculated as,

$$
S D=\sqrt{\frac{\sum_{i \in V}(E N[i]-\mu)^{2}}{|V|}}
$$

In the equation (2), EN[i] is the energy consumption of node $i, V$ is the set of sensor nodes, and $\mu$ is the average energy consumption of sensor nodes. A large variation in the SD value means that some parts of the WSN is likely to exhaust its energy sooner than other parts. A small SD value means uniform energy consumption and hence improved network lifetime. In the fig. 5, SS approach shows high variation in SD values due to energy hole problem, while for RSM, SD variation is in between SS and NBWRP. NBWRP has the lowest SD values indicating that its energy consumption distribution is far better than the other two approaches. 


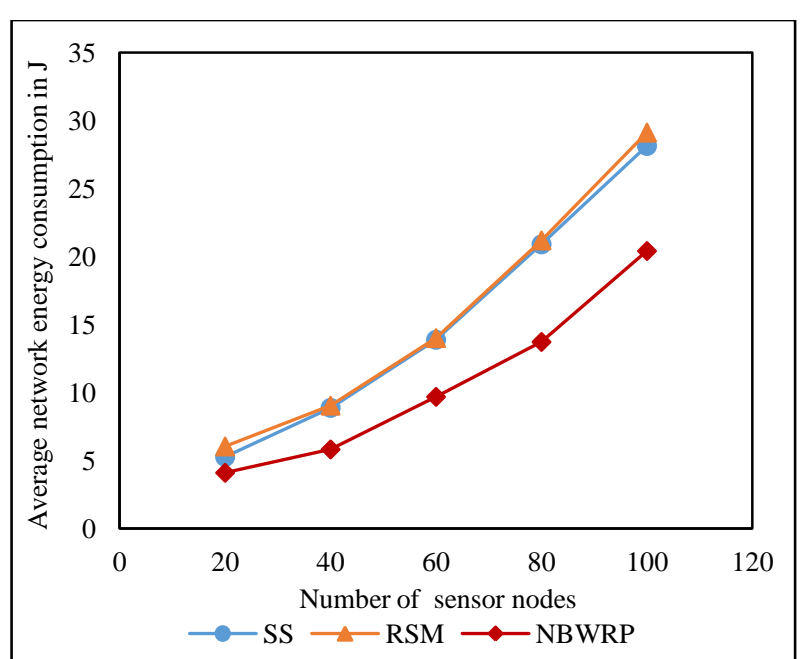

Fig. 4: Average network energy consumption for SS, RSM and NBWRP.

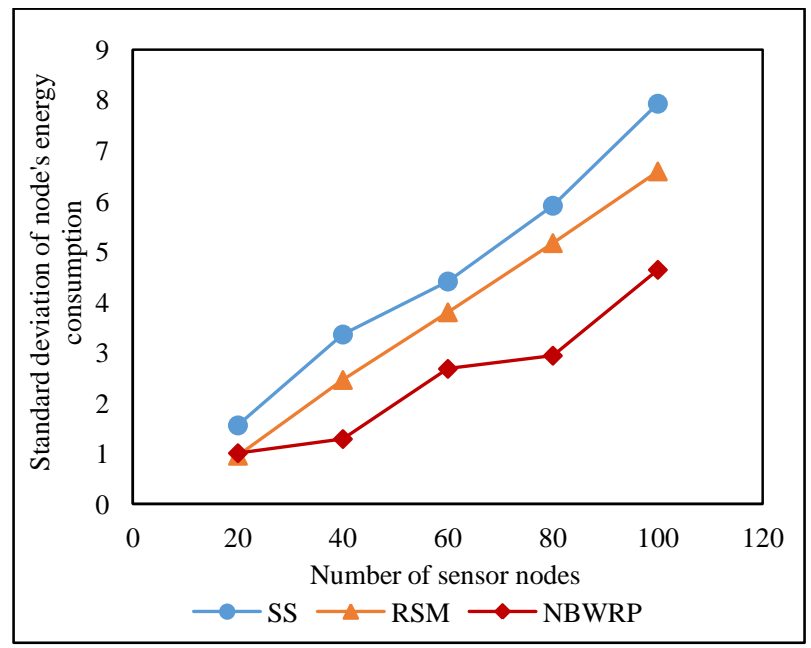

Fig. 5: Standard deviation of sensor nodes' energy consumption for SS, RSM and NBWRP.

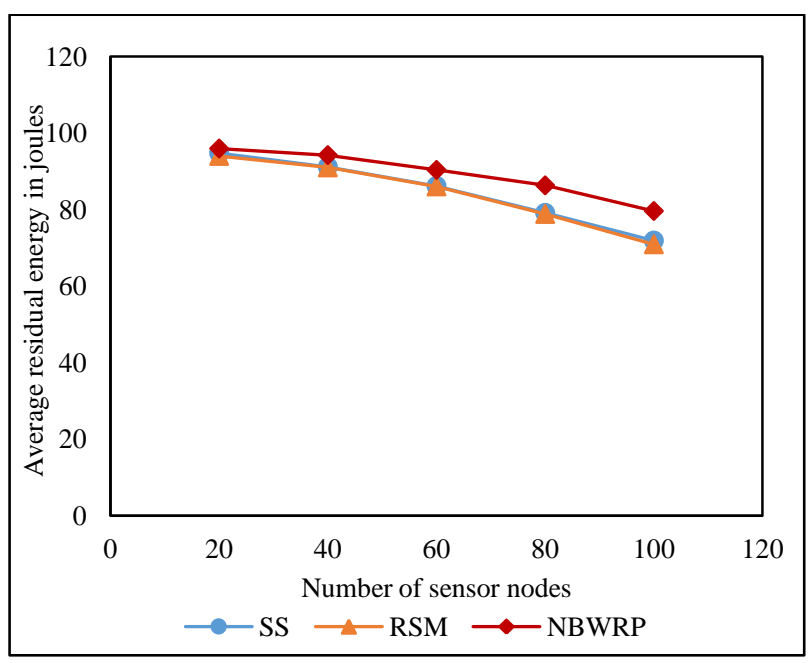

Fig. 6: Average residual energy of the network for SS, RSM and NBWRP.

Fig. 6 shows the average residual energy (in joules) of the network versus number of sensor nodes. The residual energy of NBWRP is higher for different number of nodes when compared to SS and RSM. Fig. 7 shows the network life time of SS, RSM and NBWRP versus different number of nodes. Here we define network lifetime as the time until the first node exhausts completely. Clearly NBWRP approach has higher lifetime as compared with the lifetime of the other two approaches. This is due the fact that the "energy hole" problem is effectively minimized in NBWRP approach.

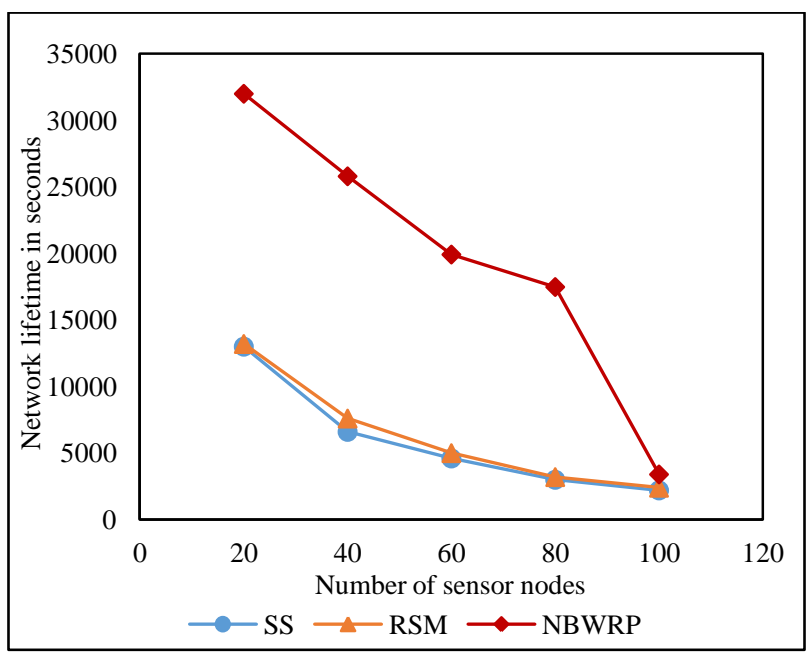

Fig. 7: Network lifetime for SS, RSM \& NBWRP.

\section{CONCLUSIONS AND FUTURE WORK}

Energy consumption using mobile sink is a critical research area of power conservation in WSN. This paper addresses the problems that arise due to "energy hole" phenomenon and uneven distribution of energy consumption in WSNs where there is a timeliness constraint on the data to be collected by the sink. We have presented a rendezvous based algorithm that attempts to minimize and uniformly distribute the energy expenditure of the network while ensuring the sink collects the sensed data within a given deadline. The algorithm enhances the lifetime of the network by designating those RPs that reside in the congested areas of the network. The results presented in section 6 show that this technique leads to better energy consumption and hence it enhances the lifetime of the whole network. We compared the performance of NBWRP with static sink and random movement strategy. NBWRP effectively minimizes the "energy hole" problem by evenly distributing energy consumption of the sensor nodes. In this paper we have considered the same deadline for all data packets. However, in some applications, sources may have different data collection deadlines due to their unique properties. In such applications, sink should plan its visiting schedule that takes all deadlines into account. Therefore as a future work we can extend this algorithm to deal with multiple deadlines. Moreover, the algorithm can also be extended to include multiple mobile sinks.

\section{ACKNOWLEDGEMENT}

The euphoria of successfully completing this research work would be incomplete without expressing our gratitude to those people who have helped us to achieve this milestone. We take this opportunity to express our deepest gratitude and appreciation to all those who helped us directly or indirectly towards the successful completion of this research work. 


\section{REFERENCES}

[1] M. A. Matin and M. M. Islam "Overview of wireless sensor network”, InTech, Croatia, 2012, 3-24.

[2] Arun A. Somasundara, Aditya Ramamoorthy and Mani B. Srivastava, "Mobile element scheduling for efficient data collection in wireless sensor networks with dynamic deadlines", Proceedings of the $25^{\text {th }}$ IEEE international real-time systems symposium (RTSS), IEEE, 2004.

[3] Tifenn Rault, Abdelmadjid Bouabdallah and Yacine Challal, "WSN lifetime optimization through controlled sink mobility and packet buffering", Global information infrastructure symposium, IEEE, Trento, October 2013.

[4] Hamidreza Salarian, Kwan-Wu Chin and Fazel Naghdy, "An energy efficient mobile sink path selection strategy for wireless sensor networks", IEEE Transactions on vehicular technology, Vol. 63, No. 5, June 2014.

[5] Guoliang Xing, Tian Wang, Zhihui Xie and Weijia Jia, "Rendezvous planning in wireless sensor networks with mobile elements", IEEE transaction on mobile computing, Vol. 7, No. 12, December 2008.

[6] X. Li, A. Nayak and Stojmenovic, "Sink mobility in wireless sensor networks", Wiley, Hoboken, NJ, USA, 2010, 153-184.

[7] Shuai Gao, Hongke Zhang and Sajal K. Das, "Efficient data collection in wireless sensor networks with pathconstrained mobile sinks", IEEE transaction on mobile computing, Vol. 10, No. 5, April 2011.

[8] Guoliang Xing, Tian Wang, Weijia Jia and Minming Li, "Rendezvous design algorithms for wireless sensor networks with a mobile base station”, ACM Press, Hong Kong, China, 2008.

[9] Khaled Almi'ani, Anastasios Viglas and Lavy Libman, "Energy-efficient data gathering with tour lengthconstrained mobile elements in wireless sensor networks", Proceedings of the $35^{\text {th }}$ IEEE Conference, LCN, Denver, CO, USA, October 2004, 582-589.

[10] E. Ekici, Y. Gu and D. Bozdag, "Mobility-based communication in wireless sensor networks," IEEE Communications Magazine, vol. 44, Jul 2006, 56-62.

[11] A. Mainwaring, D. Culler, J. Polastre, R. Szewczyk and J. Anderson, "Wireless sensor networks for habitat monitoring," in Proc. 1st ACM Int. Workshop Wireless Sens. Netw. Appl., New York, NY, USA, Sep. 2002, 8897.

[12] W. Chen, L. Chen, Z. Chen and S. Tu, "Wits: A wireless sensor network for intelligent transportation system," in Proc. 1st Int. Multi-Symp. Comput.Comput. Sci., Hangzhou, China, vol. 2, Jun. 2006.

[13] B. Sun, L. Osborne, Y. Xiao and S. Guizani, "Intrusion detection techniques in mobile ad hoc and wireless sensor networks," IEEE Wireless Communications, vol. 14 , no. 5, 2007, 56-63.
[14] T. Miyazaki, R. Kawano, Y. Endo and D. Shitara, "A sensor network for surveillance of disaster-hit region," in Proceedings of the 4th International Symposium on Wireless and Pervasive Computing, February 2009, 1-6.

[15] J. R. Polastre, Design and implementation of wireless sensor networks for habitat monitoring, M.S. thesis, University of California at Berkeley, 2003.

[16] R. Shah, S. Roy, S. Jain and W. Brunette, "Data MULEs: modeling a three-tier architecture for sparse sensor networks," in Proceedings of the First IEEE International Workshop on Sensor Network Protocols and Applications, 2003, 30-41.

[17] Y. Gu, D. Bozdag, E. Ekici, F. Ozguner and C. -G. Lee, "Partitioning based mobile element scheduling in wireless sensor networks," in Proceedings of the Second Annual IEEE Communications Society Conference on Sensor and Ad Hoc Communications and Networks(SECON), Sep 2005, 386-395.

[18] S. Gandham, M. Dawande, R. Prakash and S. Venkatesan, "Energy efficient schemes for wireless sensor networks with multiple mobile base stations," in Proceedings of IEEE Global Telecommunications Conference (GLOBECOM), Dec 2003, 377-381.

[19] Z. M. Wang, E. Melachrinoudis and C. Petrioli, "Exploiting sink mobility for maximizing sensor networks lifetime," in Proceedings of the $38^{\text {th }}$ Annual Hawaii International Conference on System Sciences (HICSS), Jan 2005.

[20] N. Christofides, "Worst-case analysis of a new heuristic for the travelling salesman problem", Graduate school of industrial administration, Carnegie-Mellon University, Technical report 388, 1976.

[21] Kansal A., Somasundara A. A., Jea D. D., Srivastava M. B. and Estrin D., "Intelligent fluid infrastructure for embedded networks", Proceedings of the 2nd International Conference on Mobile Systems, Applications, and Services (MobiSys), Boston, Massachusetts, USA, 2004, 111-124.

[22] Xing G., Wang T., Xie Z. and Jia W., "Rendezvous planning in mobility-assisted wireless sensor networks", Proceedings of the 28th IEEE International Real-Time Systems Symposium (RTSS), Tucson, Arizona, USA, 2007, 311-320

[23] Sugihara R. and Gupta R. K., "Improving the data delivery latency in sensor networks with controlled mobility", Proceedings of the 4th IEEE International Conference on Distributed Computing in Sensor Systems (DCOSS), Santorini Island, Greece, Volume 5067 of LNCS, Santorini Island, Greece, 2008, 386-399.

[24] Gu Y., Bozdag D. and Ekici E., "Mobile element based differentiated message delivery in wireless sensor networks", Proceedings of the 2006 IEEE International Symposium on a World of Wireless, Mobile and Multimedia Networks (WoWMoM). 